\title{
Réflexions sur les Relations Commerciales Internationales *
}

\author{
par Olivier Long **
}

\section{Introduction}

Parmi les risques assumés par les entreprises engagées dans le commerce international, il en est un qui échappe à leur contrôle et qu'en général elles ne peuvent pas assurer, c'est le risque des interventions gouvernementales. Par exemple, la restriction à l'importation qui ferme soudainement un marché, le relèvement d'un droit de douane, l'aide publique accordée à un concurrent, et beaucoup d'autres encore.

En période de difficultés économiques, les gouvernements sont poussés à intervenir sur les échanges commerciaux pour protéger leurs marchés intérieurs et leurs entreprises nationales. Le pire exemple d'un tel phénomène reste celui de la grande dépression des années 30 . Face à la crise économique, les pays avaient réagi en fermant leurs frontières aux produits étrangers. Ce faisant, ils avaient aggravé la crise, étouffé les économies nationales, augmenté faillites et chômage.

Cette expérience désastreuse avait montré combien il est important de maintenir les échanges aussi libres que possible, même en période de crise, et la nécessité, pour cela, de pouvoir s'appuyer sur un système commercial international. C'est avec ce double objectif à l'esprit qu'après la deuxième guerre mondiale, les pays commerçants ont créé le GATT, l'Accord général sur les tarifs douaniers et le commerce, entré en vigueur en 1948.

Le GATT est à la fois un cadre juridique gouvernant la conduite des relations commerciales, un forum de négociation pour la libéralisation du commerce et un organe de conciliation et de règlement des différends entre les pays membres. C'est un instrument souple qui ne cesse de faire l'objet d'adaptations en fonction de l'évolution politique et économique.

\section{Etat des relations commerclales}

Depuis 1948 , bien du chemin a été parcouru. Le cadre juridique et institutionnel du GATT s'est élargi et renforcé. Simultanément, le volume du commerce international a augmenté de façon spectaculaire.

* Conférence présentée à l'occasion de l'Assemblée Générale de l'Association Internationale pour l'Etude de l'Economie de l'Assurance, tenue à Genève en juillet 1980.

** Directeur Général du G.A.T.T. (General Agreement on Tariffs and Trade), Genève. 
Le nombre des pays participants a passé de 23 au départ à 88 actuellement, plus 31 pays qui appliquent le GATT de facto en attendant d'avoir définitivement arrêté leur politique commerciale. Entre eux, ils font plus des $4 / 5$ du commerce mondial.

Le champ d'action des dispositions de l'Accord général s'est aussi élargi en fonction des besoins nouveaux, afin d'assurer une surveillance plus étendue des mesures gouvernementales qui affectent les échanges de marchandises.

L'élargissement du champ d'action du GATT se fait par la voie de négociations entre les pays participants, qui aboutissent à des accords juridiquement contraignants. C'est-à-dire qu'ils donnent aux gouvernements des droits et leur imposent des obligations dont le but est de mettre les entreprises privées à l'abri des risques causés par les interventions de l'Etat dans les conditions des échanges de marchandises.

Au titre de la libéralisation des échanges, le principe clé est celui de la clause de la nation la plus favorisée. Tout avantage accordé par un signataire à un autre signataire est automatiquement étendu à tous les autres pays membres du GATT. En multilatéralisant ainsi les relations commerciales, la clause de la nation la plus favorisée a un effet multiplicateur sur les échanges.

La libéralisation, c'est aussi l'abaissement progressif des droits de douane. Depuis sa création, le GATT a mené à bien plus d'une demi-douzaine de négociations tarifaires, conduisant chacune à une réduction des droits de douane. Ces réductions ont chaque fois été consolidées, c'est-à-dire que les pays qui les avaient consenties se sont engagés formellement à ne pas relever les droits ainsi abaissés. Les droits de douane ont donc perdu progressivement beaucoup de leur importance en tant qu'instrument de protection. D'autre part, la consolidation des abaissements tarifaires engendre un climat de sécurité qui ouvre des débouchés stables et favorise les investissements. Bref, stimule l'économie tout entière.

Les droits de douane ne sont pas les seuls obstables aux échanges de marchandises, loin de là. C'est pourquoi le GATT avait, dès ses débuts, porté son effort sur le démantèlement des restrictions quantitatives à l'importation. C'est pourquoi aussi, plus récemment, le Tokyo Round de Négociations Commerciales Multilatérales s'est attaqué aux mesures non tarifaires qui affectent le commerce international.

Le succès de cette négociation à la fin de l'année 1979 permet une relance de la libéralisation des échanges, renforce le cadre juridique qui gouverne la conduite des relations commerciales et offre un traitement plus favorable aux pays en voie de développement.

C'est ainsi qu'a commencé une nouvelle phase d'abaissements des droits de douane d'environ $35 \%$, étalée sur huit ans. (La quatrième tranche de réductions est en vigueur depuis le $1^{\text {er }}$ janvier 1983.)

C'est ainsi que de nombreux accords ont vu le jour, qui établissent de nouvelles règles en matière non tarifaire. Je me contente de les énumérer ici sans chercher à entrer dans les détails :

- Accord sur les subventions et droits compensateurs;

- Accord sur les droits anti-dumping ; 
- Accord sur les obstacles techniques au commerce, c'est-à-dire essentiellement sur les normes ;

- Accord sur l'établissement de la valeur en douane ;

- Accord sur les marchés publics, c'est-à-dire sur les achats gouvernementaux, qui ouvrira à la concurrence internationale pour quelque 30 milliards de dollars de commerce.

Ces accords sont tous en vigueur.

Les plus importantes mesures non tarifaires qui affectent le commerce vont donc désormais être acheminés sur la voie de la libéralisation. Car tous ces accords fixent des règles et des lignes directrices pour que les mesures non tarifaires cessent d'entraver les échanges.

Il est donc permis de constater qu'à la suite du succès du Tokyo Round, il y aura plus de liberté, plus de transparence et plus de certitude dans les conditions faites aux échanges de marchandises.

Quant au commerce international, depuis 1948 - entrée en vigueur du GATT le volume des échanges de marchandises a été multiplié par neuf.

Jusqu'en 1974, on a vu une croissance quasi ininterrompue, à la moyenne annuelle de $81 / 2 \%$ du volume du commerce international. Croissance stimulée essentiellement par le développement des échanges de produits manufacturés entre pays industrialisés.

Depuis la crise de 1974 , cette croissance moyenne a fléchi. Elle a été de l'ordre de $4 \frac{1}{2} \%$ par an. Il est important de relever que, dans cette récente période, ce ne sont plus tellement les échanges de produits manufacturés entre pays industrialisés qui stimulent la croissance du commerce. Ce sont les exportations de produits manufacturés vers les pays en développement, et pas seulement vers les pays producteurs de pétrole.

En 1980, dans la sixième année de crise économique, il est frappant de constater la remarquable résistance du commerce international aux perturbations économiques et politiques.

La raison de ce phénomène, c'est probablement que le commerce international est le fait de l'initiative privée, de l'activité des entrepreneurs, tandis que la monnaie et la politique conjoncturelle sont le fait des pouvoirs publics et se trouvent, par conséquent, soumis plus directement aux contraintes politiques nationales.

En fait, malgré les pressions qu'il a subies au cours des ces dernières années, le cadre juridique et institutionnel du commerce international non seulement a tenu, mais sort renforcé par le succès du Tokyo Round. Certes, la croissance du commerce est moins brillante que précédemment. Toutefois, les échanges de marchandises, eux aussi, ont résisté et continuent même de croître, en dépit de pressions protectionnistes très sérieuses, et même de mesures protectionnistes prises dans certains secteurs sensibles. En 1979 , le volume du commerce a augmenté de $6 \%$ par rapport à l'année précédente, soit le double de la croissance de la production mondiale. (Depuis lors, du fait de l'aggravation de la récession économique et des mesures de protection qui en résultent, le volume du commerce international a pratiquement cessé de croître.) 


\section{Perspectives des relations commerclales}

Le commerce international est un moteur puissant de la croissance économique, mais il est aussi très influencé par les autres facteurs économiques, pour lesquels les perspectives restent sombres. Tous les maux dont notre monde est frappé vous sont bien connus. Il est donc inutile de les rappeler ici.

Comment le commerce international peut-il évoluer dans ces conditions ?

Entre les pays industrialisés, bien que l'imbrication de leurs échanges commerciaux soit déjà très grande, les possibilités de spécialisation dans leurs échanges sont loin d'être épuisées. En outre, ces pays ont une longue expérience de la coopération et de l'intégration. Ils se montrent capables de surmonter leurs disputes (Etats-Unis / CEE / Japon) (acier, fibres artificielles, automobiles).

Sans compter sur des développements spectaculaires, il est permis d'envisager la poursuite d'une croissance modeste des échanges entre ces pays.

Dans le commerce avec les pays à économie socialiste, le potentiel de croissance est théoriquement grand, malgré le ralentissement qui s'est produit au cours des récentes années. Les pays socialistes de l'Est européen sont très désireux de développer leur commerce avec l'Occident. Ils ont besoin d'acquérir des biens de production à l'Ouest.

Toutefois, si le potentiel existe, la réalisation est freinée par le peu d'intérêt des pays de l'Ouest à augmenter leurs achats à l'Est et leur crainte de la concurrence de produits à bas prix.

De plus, l'endettement des pays de l'Est est considérable.

A cause de ces freins, financiers d'une part, et commerciaux de l'autre, il ne faut pas s'attendre à une croissance notable du commerce Est/Ouest.

Quant aux pays de l'OPEP, ils offrent des marchés avec des possibilités de très forte croissance. Toutefois, des expériences récentes (pas seulement celle de l'Iran) montrent une expansion commerciale irrégulière, parce que sujette à des accidents de parcours d'une sorte ou de l'autre. Des goulets d'étranglement de types divers risquent de restreindre temporairement l'augmentation de leurs importations. Il ne faut donc pas s'attendre que le taux de croissance de leurs marchés soit aussi rapide dorénavant qu'il ne l'a été après la première hausse du prix du pétrole, au milieu des années 70 .

Le défi posé par les pays producteurs de pétrole au commerce mondial n'est pas tellement celui de l'évolution de leur propre capacité d'importations. Le problème fondamental est de savoir comment l'énorme déplacement de pouvoir d'achat qui a lieu dans leur direction sera redéployé ; c'est de savoir comment les autres pays pourront ajuster leurs économies aux perturbations et à l'incertitude du marché de l'énergie, et s'il y aura place pour un arrangement à long terme sur les approvisionnements et le prix du pétrole.

Quant aux pays en développement non producteurs de pétrole, leur potentiel d'importation est évidemment énorme puisque leurs besoins sont quasiment illimités. Mais, comment et dans quelle mesure ce potentiel sera-t-il réalisé ? En d'autres termes, ces pays pourront-ils augmenter suffisamment leurs exportations pour gagner de quoi importer ce dont ils ont besoin et faire face en même temps au service de leur dette ? 
Or, en 1980 , le déficit en compte courant des pays en voie de développement non producteurs de pétrole sera de l'ordre de 68 milliards de dollars. (Il a augmenté depuis lors.) En outre, il y a dans les pays industrialisés de fortes pressions protectionnistes contre les importations de produits manufacturés en provenance des pays en développement.

En ce qui concerne l'endettement des pays en voie de développement, il est considéré par l'ensemble de la communauté financière internationale - FMI, Banque Mondiale, Banques Privées - comme le problème le plus urgent dans les relations Nord/Sud. Pour ma part, j'ajouterai seulement que ce problème affecte directement les perspectives du commerce international. En effet, au cours des récentes années, la forte importation dans les pays en voie de développement de produits manufacturés en provenance des pays industrialisés a été largement financée par le recours aux emprunts. Ce rythme élevé d'importation pourra-t-il se poursuivre, étant donné le problème aigu du financement posé aux pays en voie de développement par les nouvelles hausses du prix du pétrole?

Par conséquent, du côté commercial, il est essentiel que les pays en voie de développement puissent augmenter leurs recettes d'exportation et trouvent un accès plus libre pour leurs produits manufacturés dans les marchés des pays industrialisés.

En fait, l'avenir de la croissance du commerce international passe par une plus grande ouverture des pays industrialisés aux produits des pays en voie de développement. Cela est nécessaire afin que le potentiel d'importation des pays en voie de développement puisse se déployer pour le plus grand bien des exportations des pays industrialisés et, par conséquent, de leurs économies. Ce n'est pas seulement une question d'obligation morale, ni de sagesse politique, mais tout simplement d'intérêt bien compris. N'oublions pas que la croissance relativement satisfaisante du volume du commerce mondial au cours de la seconde moitié des années 70 provient dans une large mesure du niveau élevé des importations dans les pays en voie de développement, producteurs et non producteurs de pétrole.

\section{L'élargissement de l'action du GATT au secteur des services ?}

Avant de répondre à cette question il faut, tout d'abord, préciser que le GATT est une institution pragmatique. Son approche des problèmes n'est ni politique, ni ideologique, mais essentiellement pratique.

Cette approche consiste à commencer par identifier les mesures restrictives, non pas de façon théorique, mais par la voie de la discussion entre les gouvernements intéressés.

Ensuite, le travail au GATT consiste à classer les mesures restrictives ainsi identifiées, puis à déterminer si elles sont négociables, et de quelle manière.

C'est de cette façon que le GATT a procédé avec les mesures non tarifaires qui affectent le commerce, et qui font maintenant l'objet des accords qui sont en vigueur depuis le début 1980 . C'est un processus qui a duré douze ans.

D'autre part, le GATT ne cherche pas à se faire donner des tâches supplémentaires. En revanche, il s'est toujours montré assez flexible et adaptable pour s'acquitter 
des tâches nouvelles dont les pays participants l'ont chargé. Encore une fois, les mesures non tarifaires en sont l'exemple le plus récent et le plus important.

Je peux aussi noter qu'une rencontre de caractère informel, portant sur la question des restrictions aux activités du secteur des services, a réuni récemment des professionnels de ces secteurs, des fonctionnaires gouvernementaux et des économistes venant des Etats-Unis et de l'Europe.

$\mathrm{Au}$ cours de cette réunion, un certain consensus s'est dégagé sur les points suivants :

a) L'importance grandissante du secteur des services dans l'ensemble de l'activité économique provoquera un intérêt croissant pour les échanges internationaux de services et pour les politiques gouvernementales qui affectent ces services.

b) Dans un nombre croissant de secteurs des services, on commence à croire, en particulier aux Etats-Unis, que bien des problèmes rencontrés dans les affaires faites avec l'étranger, sont de caractère général plutôt que particuliers aux assurances, aux communications ou à d'autres secteurs de service encore.

c) Une libéralisation du commerce des services, aussi large que politiquement possible, est un objectif approprié à long terme. Par libéralisation, on entend que le maximum de liberté soit donnée aux individus pour acheter des services auprès des entreprises de leur choix, sans que les frontières nationales constituent un obstacle.

d) Les principes directeurs, les méthodes, les analyses techniques employées dans le domaine des échanges de marchandises semblent pouvoir être transposés sans de trop grandes modifications au secteur des services.

e) Un travail préparatoire considérable de recherche, d'analyse et d'explications auprès des milieux d'affaires et des responsables politiques serait indispensable avant qu'il ne devienne possible de progresser dans la réduction des obstacles aux échanges internationaux de services, surtout si l'on envisage des progrès dans un cadre multilatéral.

(La Conférence ministérielle du GATT, en novembre 1982, a montré qu'il existe encore de larges divergences de vues entre les gouvernements sur l'opportunité d'entamer des négotiations sur les services.)

\section{Conclusion}

Il y a encore de bonnes possibilités d'expansion du commerce international, surtout dans le cadre Nord/Sud. En outre, l'expérience passée, y compris celle de la présente récession, montre que la croissance commerciale a presque toujours dépassé les prévisions des experts, grâce à l'initiative et à la capacité d'adaptation et d'innovation de l'entreprise privée.

De plus, le système commercial international fondé sur le GATT est bien équipé pour gérer la crise. A la condition, cependant, que les gouvernements aient la capacité politique de s'en servir de façon constructive.

Toutefois, tant que l'économie mondiale demeurera déprimée et que les gouvernements ne sauront pas mener des politiques de nature à faciliter des investissements productifs de la part des entreprises privées, les perspectives commerciales des années 80 resteront peu encourageantes. 